\title{
Profile of factors influencing academic motivation among grade 6 and 7 learners at a state school
}

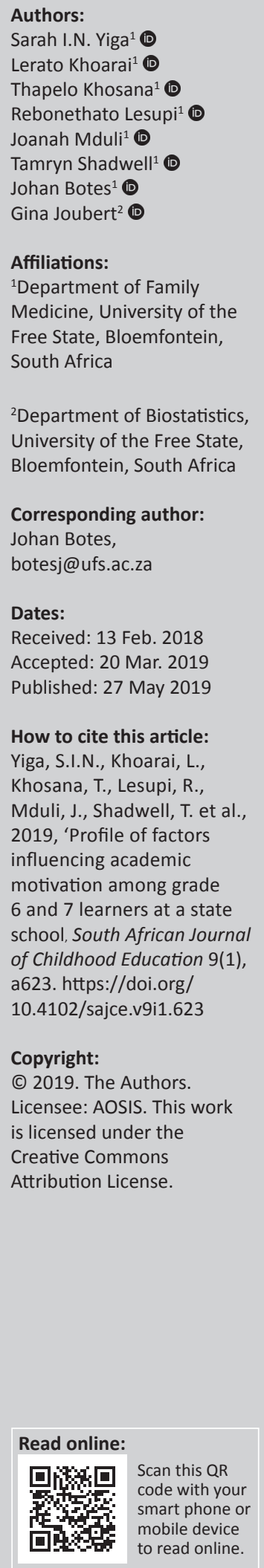

Authors:

Rebonethato Lesupi ${ }^{1}$

Johan Botes ${ }^{1}$ (1)

Affiliations:

${ }^{1}$ Department of Family

edicine, University of the

South Africa

${ }^{2}$ Department of Biostatistics, University of the Free State,

Corresponding author:

Johan Botes,

Dates:

Accepted: 20 Mar. 2019

article:

Yiga, S.I.N., Khoarai, L.,

2019, 'Profile of factors

influencing academic

school, South African Journal

of Childhood Education 9(1),

a623. https://doi.org/

Licensee: AOSIS. This work

is licensed under the

Creative Commons

Attribution License.

mobile device
to read online.
Background: Academic achievement is influenced by a system of internal and external stimuli. Internal stimuli include interest, willingness and academic motivation. In South Africa, efforts to improve the quality of education have mostly focused on the provision of physical resources rather than emotional resources.

Aim: The aim of this study was to determine the profile of four factors, namely, teacher style, role models, home environment and peer influence that can influence the academic motivation of grade 6 and 7 learners.

Setting: A parallel-medium primary state school in an urban part of Bloemfontein, Free State.

Methods: This was a cross-sectional observational study. Data were collected using an anonymous, self-administered questionnaire completed by the learners. The questions captured demographic data and measured the four categories of factors.

Results: Overall, 115 out of 202 learners participated (response rate 56.9\%). Almost all learners felt that their teachers encouraged them to do their best $(96.5 \%)$, but $61.3 \%$ felt they could not confide in their teachers with personal problems. Most learners had a role model $(93.8 \%)$, and $70.9 \%$ expressed that the role model's hard work was the reason for their admiration. Most learners felt that they were surrounded by supportive people $(83.0 \%)$ and had a good study environment at home $(80.5 \%)$. The majority of learners had a group of friends with whom they felt they belong $(90.3 \%)$, and they could confide in their best friends with personal problems (61.6\%).

Conclusion: Teacher style, peers and home environment scored high as important factors for academic motivation.

Keywords: academic motivation; factors; teacher; role model; home environment; peer.

\section{Introduction}

Academic achievement is influenced by a complicated system of internal and external stimuli. All these factors interplay, and the weight of their individual influences differs between countries, communities, demographic groups and individuals (Allison 2010; McCoy, Wolf \& Godfrey 2014). Internal stimuli include academic motivation, interest in the subject matter and the learner's willingness to participate (Komarraju, Karau \& Schmeck 2009; McCoy et al. 2014). The main external stimuli are the academic platform, teachers, parents and peers, and the expectations and attitude of the immediate community (Allison 2010; Chen 2008; McCoy et al. 2014; Schapps 2003).

Academic motivation can be described as the self-determination to succeed in learning. This is formed by the psychological and personality traits of the individual, which, in turn, is influenced by a number of external factors (Komarraju et al. 2009). Motivation, as a concept, consists of a spectrum with two poles called 'intrinsic motivation' and 'amotivation'. Internal and external factors stimulate the learner, and the position of the learner in the spectrum is determined by the way the learner reacts to these factors. A learner, who is internally self-determined to succeed, will be closer to the intrinsic pole. The more external influences are required, the closer the learner is to the middle of the spectrum (called 'extrinsic motivation'), while those who show no interest and cannot be influenced internally or externally are on the amotivation side (Komarraju et al. 2009).

We conducted a review of the literature in an attempt to find influencing factors that may play a role in the learner's academic motivation. The following list of possible factors was formulated: teacher style, role models, home environment and peer influence. 
Most teachers aspire to offer an influential learning environment and the best possible opportunities for learners. Unfortunately, teachers face many obstacles in the form of standardised curricula, specifically set outcomes and limited resources provided by the school system (Allison 2010). Thoonen et al. (2011) investigated the correlation between the teacher's influence and learners' learning motivation. They found a definite positive effect in all aspects, except in process-orientated educating where learners showed a decrease in motivation. Schapps (2003) emphasised the importance of the school environment where learners become motivated when they feel safe and have a sense of belonging. When learners experience a positive environment, they eventually become part of the culture and contribute to the school and learning community.

Role models also play a part in motivation. Morgenroth, Ryan and Peters (2015) summarised a role model's functions as a model for behaviour, depiction of what could be achieved and as inspiration. This usually occurs when the person can identify with the role model in some manner and share similar goals. Lockwood, Jordan and Kunda (2002) explained that both positive and negative role models could positively guide a learner on how to - or not to - behave and be successful.

The home environment, especially parental figures, is an important factor in motivating learners to be successful academically. In fact, Harris and Goodall (2008) found that only if parents or guardians are more involved in the learning process, could a learner be expected to improve on his academic performance. Despite the busy lives of parents today, the learning process requires time and commitment for the child to be successful.

Peers could be a positive influence on learners' motivation, but could just as easily have a negative impact. The environment, culture and social interaction between peers could help a learner experience belonging and learning could be boosted, but a negative effect could occur if a learner struggles to adapt to the group dynamic (Chen 2008).

South Africa achieves excellent school enrolment of more than $98 \%$ for children between the ages of 7 and 15 . However, only one in two learners enrolled in grade 1 will eventually pass matric (Modisaotsile 2012).

So far, efforts to improve the quality of education have focused strongly on the provision of physical resources rather than emotional resources or motivation. This approach is apparent in the Department of Basic Education's Strategic Plan 2011-2014 and their annual reports (Department of Basic Education [DBE] 2011). These documents emphasise the provision of textbooks on time, training more teachers, improving school infrastructure and developing a better curriculum (DBE 2011). These are all essential, but there is little focus on the learners' willingness to learn.
The aim of this study was to determine the profile of factors, namely, teacher style, role models, home environment and peer influence, that can influence the academic motivation of grade 6 and 7 learners at a primary state school in Bloemfontein, South Africa.

\section{Research methods and design Study design and setting}

This was a cross-sectional observational study. A parallelmedium primary state school in an urban part of Bloemfontein was chosen as study site. This is a co-educational and feepaying school with good physical resources.

\section{Study population and sampling strategy}

The study population consisted of all grade 6 and 7 learners enrolled at the school in 2013. All learners willing to participate in the study were included in the sample.

\section{Data collection}

An anonymous, self-administered questionnaire was designed by the authors. Mainly closed questions were formulated on the pre-selected factors according to the relevant literature. The questionnaire was available in English, Afrikaans and Sesotho. The learners could select the language in which they wanted to complete the questionnaire. The questions measured demographic data and the four categories of factors: teacher style, role models, home environment and peer influence. Home environment included questions on parents or guardians, physical environment and socioeconomic status.

Questions were either Yes/No or multiple choices. Weights were pre-set for each possible answer, ranging from ' 1 ' given for a definite positive answer and ' 0 ' for no influence on their motivation. The teachers were asked to explain the questionnaire to the learners, but were asked not to be involved in the completion thereof. Grade 6 and 7 teachers handed out the questionnaires to the learners. The completed questionnaires were collected by the student researchers on two occasions.

\section{Pilot study}

The pilot study was conducted at another similar primary school in Bloemfontein on 20 grade 6 and 7 learners. The structuring of the questions and options provided in the questionnaire were adjusted according to feedback. Data from the pilot study were not included in the main study.

\section{Data analysis}

The data were analysed by the Department of Biostatistics, Faculty of Health Sciences, University of the Free State. Each answer had a unique numerical code, which was scored according to the memo, based on the literature. Each category was scored separately. If more than half of the questions in 
a category were answered, the score for that category was calculated using the completed questions. Based on these category marks, the learners were divided into three motivation strata (low: $0 \%-33 \%$, intermediate: $34 \%-66 \%$ and high: $67 \%-100 \%$ ) for each of the four categories.

\section{Ethical considerations}

The study was approved by the Ethics Committee of the Faculty of Health Sciences, University of the Free State (STUD 28/2013). Permission to conduct the study was obtained from the Head of the Free State Department of Education and the Education District Director of the Motheo Region. Signed permission was obtained from the school principal, teachers, participating learners and their parents. All data were handled confidentially, and no identifiable information was recorded on the questionnaire.

\section{Results}

Of the 202 learners invited to participate, 115 (response rate $56.9 \%$ ) consented and participated. The vast majority (93.9\%) of the participants completed their questionnaires in English, even though only $18.3 \%$ had English as their home language. The highest percentage $(44.4 \%)$ of the learners indicated that they spoke Sesotho at home, 9.3\% indicated Afrikaans, while other, mainly African languages, made up the remaining $27.8 \%$. Boys were slightly in the majority $(56.5 \%)$, most of the participants were in grade $6(67.8 \%)$ and the median age was 12 years. The majority of the learners came to school by private car $(60.9 \%)$ or public transport $(20.0 \%)$. For $82.6 \%$ of the learners, travelling time to school was $30 \mathrm{~min}$ or less.

\section{Teacher style}

Participating learners stated that teachers created a comfortable environment for group work (81.4\%). They felt teachers explained the incorrect answers sufficiently $(88.5 \%)$, enough time was given to work independently (78.8\%) and teachers were willing to be corrected when they were wrong $(82.3 \%)$. Teachers encouraged the learners to do their best (96.5\%), but $61.3 \%$ of the learners felt they could not confide in teachers with personal problems.

\section{Role models}

The majority of the learners had a role model (93.8\%), and $70.9 \%$ of the learners expressed that hard work was the reason for their admiration. The learners stated that they were more influenced by a positive role model ( $83.2 \%)$ than a negative role model; though those who indicated they have a negative model $(67.6 \%)$ felt there was still an opportunity to learn $(92.0 \%)$.

\section{Home environment}

Most of the learners (80.5\%) felt that they had a good study environment at home and were surrounded by supportive people (83.0\%). Approximately all (93.8\%) felt that hard work determines success, more so than background. Only 32.7\% felt those from a wealthy background have more opportunities. The learners had someone at home to help with their homework (82.3\%). They also had someone at home to talk to about what happened at school (76.6\%) and encourage them to work hard $(96.8 \%)$.

\section{Peer influence}

The majority of learners had a group of friends with whom they felt they belong (90.3\%), and they could confide in their best friends with personal problems (61.6\%). The learners indicated that they were willing to differ from their friends when their best friends disagreed with them about important decisions (60.0\%). Regarding encouragement, 52.2\% stated their friends motivated them when needed. When the best friend outperformed them, $68.1 \%$ felt encouraged to work harder.

In Table 1, the answers are categorised and scored to give an overview of the important factors influencing learning.

More than $60 \%$ of learners fell into the high motivational stratum for teacher style (61.4\%), home environment (66.0\%) and peer influence $(71.1 \%)$. Only $20.0 \%$ of the learners scored high for motivation by role models. A greater percentage of girls scored high for motivation by their teacher $(66.0 \%)$ and peers $(74.0 \%)$, compared with the boys. Both boys $(66.1 \%)$ and girls $(66.0 \%)$ scored equally high in motivation for home environment.

\section{Discussion}

Grade 6 and 7 learners at this Bloemfontein primary state school are motivated by their teachers and peers and have academically supportive home environments. The 'peers' category had the highest percentage of learners in the high motivational stratum, closely followed by home environment and teacher influence. Genders did not differ markedly. This study did not assess the association between these factors and learners' academic performance.

TABLE 1: Scores per motivation strata for each category.

\begin{tabular}{lccc}
\hline Category & \multicolumn{3}{c}{ Motivation strata } \\
\cline { 2 - 4 } Teacher & Low (\%) & Intermediate (\%) & High (\%) \\
Boys $(n=64)$ & 3.1 & 39.1 & 57.8 \\
Girls $(n=50)$ & 2.0 & 32.0 & 66.0 \\
Total $(n=114)$ & 2.6 & 36.0 & 61.4 \\
Role models & & & \\
Boys $(n=65)$ & 21.5 & 60.0 & 18.5 \\
Girls $(n=50)$ & 30.0 & 48.0 & 22.0 \\
Total $(n=115)$ & 25.2 & 54.8 & 20.0 \\
Home environment & & & \\
Boys $(n=63)$ & 7.7 & 26.2 & 66.1 \\
Girls $(n=50)$ & 6.0 & 28.0 & 66.0 \\
Total $(n=113)$ & 7.0 & 27.0 & 66.0 \\
Peers & & & \\
Boys $(n=64)$ & 0 & 31.3 & 68.8 \\
Girls $(n=50)$ & 0 & 26.0 & 74.0 \\
Total $(n=114)$ & 0 & 29.0 & 71.1 \\
\hline
\end{tabular}


However, this school outperformed national and provincial figures on Annual National Assessments: literacy 60.0\% compared to $42.8 \%$ nationally and $52.2 \%$ provincial, and numeracy $48.0 \%$ compared to $26.7 \%$ national and $28.4 \%$ provincial.

Most learners at this primary state school responded positively regarding teacher aspects, despite the constraints mentioned by Allison (2010). Teachers' emotional support role was less well fulfilled. Just over a third of the learners would confide in their teacher.

Almost all learners had positive role models. Of these, most stated that the role model's hard work was the reason for their admiration. Two-thirds of learners indicated the presence of a negative role model in their lives. A negative role model is not necessarily an impediment to motivation (Lockwood et al. 2002). It is still an opportunity to learn. It is important to select positive characteristics to emulate. Admiration and independence should co-exist. Bricheno and Thornton (2007) found very little evidence of learners considering teachers as role models. Most learners had role models in the home environment, extended family or community, as was the case in this study as well.

Home environment plays an important role in the academic performance of learners, especially if there is an adult available for academic assistance and emotional support when needed (Chiu \& Xihua 2008; Harris \& Goodall 2008).

It is positive to see that almost all the learners felt they belonged to a group of friends. The learners felt positively influenced by their friends, and healthy competition between friends to perform academically was evident.

\section{Study limitations}

The response rate was low because of multiple consent levels required for participation. The validity of the questionnaire depended on the honesty of the learners' answers. Repeatability was not determined as the questionnaire was only administered once. The completed questionnaires were collected on two separate days in late 2013, 5 weeks apart. Ideally, the questionnaires should have been completed and collected on a single day to prevent the learners discussing them. Researchers were not present while the learners completed the questionnaire.

Being restricted to one school also meant we could not assess other variables, which could influence motivation and academic performance such as school type (urban, rural, farm, hospital), gender composition (co-educational or single sex) and level of affluence (private, public fee-paying and public no-fee).

\section{Conclusion}

This study has attempted to measure and quantify the factors that are described in the literature as important for academic motivation. At this parallel-medium co-educational fee-paying primary state school in an urban part of Bloemfontein, learners scored high on motivation by teacher style, peers and home environment. 'Role models' was the category in which the largest percentage of children scored low.

\section{Recommendations}

This project did not intend to provide representative data, but rather to open the discussion of possible influences from external factors on the learner's academic motivation. An association between these factors and learners' motivation, leading to actual academic performance, should be investigated in a range of schools covering different school types and socio-economic levels.

\section{Acknowledgements}

The authors thank Ms T. Mulder, medical editor, School of Medicine, University of the Free State, for technical and editorial preparation of the article.

\section{Competing interests}

The authors declare that they have no financial or personal relationships that may have inappropriately influenced them in writing this article.

\section{Authors' contributions}

L.K., T.K., R.L., J.M. and T.S. developed the protocol, performed the data collection and did the initial write-up of this study. S.I.N.Y. was the supervisor of this study; suggested the concept; and assisted with the protocol development, data collection and interpretation, and write-up of the study. J.B. assisted with the protocol submission, data interpretation and write-up of the article. G.J. assisted with the planning, performed data analysis and assisted with the interpretation and write-up of the article.

\section{Funding information}

This research received no specific grant from any funding agency in the public, commercial or not-for-profit sectors.

\section{Data availability statement}

Data sharing is not applicable to this article as no new data were created or analysed in this study.

\section{Disclaimer}

The views expressed in this article are the authors' own and not an official position of the institution or funder.

\section{References}

Allison, E.B., 2010, 'Pedagogy - How is it influenced in primary schools? A comparative study of literature about pedagogical influences in primary schools in England and Poland, with a focus on English primary schools', Education 3-13 38(1), 55-67. https://doi.org/10.1080/03004270903099819

Bricheno, P. \& Thornton, M.E., 2007, 'Role model, hero or champion? Children's views concerning role models', Educational Research 49(4), 383-396. https://doi.org/ 10.1080/00131880701717230 
Chen, J.J.-L., 2008, 'Grade-level differences: Relations of parental, teacher and peer support to academic engagement and achievement among Hong Kong students',
School Psychology International 29(2), 183-198. https://doi.org/10.1177/01430 34308090059

Chiu, M.M. \& Xihua, Z., 2008, 'Family and motivation effects on mathematics achievement: Analyses of students in 41 countries', Learning and Instruction 18(4) 321-336. https://doi.org/10.1016/j.learninstruc.2007.06.003

Department of Basic Education, Republic of South Africa, 2011, Strategic Plan 20112014, viewed 31 January 2018, from https://www.education.gov.za/Portals/0/ Documents/Reports/DBE\%20StratPlan\%202011-2014.pdf?ver=2015-01-30111357-713.

Harris, A. \& Goodall, J., 2008, 'Do parents know they matter? Engaging all parents in learning', Educational Research 50(3), 277-289. https://doi.org/10.1080/00131 880802309424

Komarraju, M., Karau, S.J. \& Schmeck, R.R., 2009, 'Role of the Big Five personality traits in predicting college students' academic motivation and achievement', Learning and Individual Differences 19(1), 47-52. https://doi.org/10.1016/j.lindif. 2008.07.001
Lockwood, P., Jordan, C.H. \& Kunda, Z., 2002, 'Motivation by positive or negative role models: Regulatory focus determines who will best inspire us', Journal of Personality models: Regulatory focus determines who will best inspire us', Journal of Personality

McCoy, D.C., Wolf, S. \& Godfrey, E.B., 2014, 'Student motivation for learning in Ghana: Relationships with caregivers' values toward education, attendance, and academic achievement', School Psychology International 35(3), 294-308. https://doi.org/ 10.1177/0143034313508055

Modisaotsile, B.M., 2012, The failing standard of basic education in South Africa, viewed 31 January 2018, from http://www.ai.org.za/wp-content/uploads/downloads/ 2012/03/No.-72.The-Failing-Standard-of-Basic-Education-in-South-Africa1.pdf.

Morgenroth, T., Ryan, M.K. \& Peters, K., 2015, 'The motivational theory of role modeling: How role models influence role aspirants' goals', Review of General Psychology 19(4), 465-483. https://doi.org/10.1037/gpr0000059

Schapps, E., 2003, The role of supportive school environments in promoting academic success, California Department of Education Press, Sacramento, CA.

Thoonen, E.E., Sleegers, P.J., Peetsma, T.T. \& Oort, F.J., 2011, 'Can teachers motivate students to learn?', Educational Studies 37(3), 345-360. https://doi.org/10.1080/ 03055698.2010.507008 